\title{
ACSNANO
}

\section{Opportunities and Challenges in DNA-Hybrid Nanomaterials}

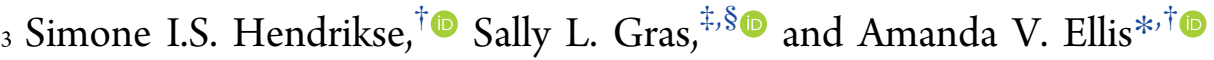 \\ $4{ }^{\dagger}$ School of Chemical and Biomedical Engineering, Melbourne School of Engineering, ${ }^{\ddagger}$ The ARC Dairy Innovation Hub, Department \\ 5 of Chemical Engineering, and ${ }^{\S}$ The Bio21 Molecular Science and Biotechnology Institute, The University of Melbourne, Parkville, \\ 6 Victoria 3010, Australia
}

\begin{abstract}
Nature has inspired the development of many life-like materials. Although still simplistic, key biological functionalities have been incorporated, enabling a wide variety of applications. DNA-based systems, in particular, show high promise due to their ability to merge specific Watson-Crick base pairing with unique properties that are also programmable, scalable, or dynamic. By combining the fields of DNA-based covalent polymers, DNA origami, and DNA-functionalized supramolecular polymers, new frontiers in next-generation DNA-based hybrid materials that can outperform current bioartificial systems will be realized. Many challenges must still be overcome before this emerging technology can be materialized.
\end{abstract}

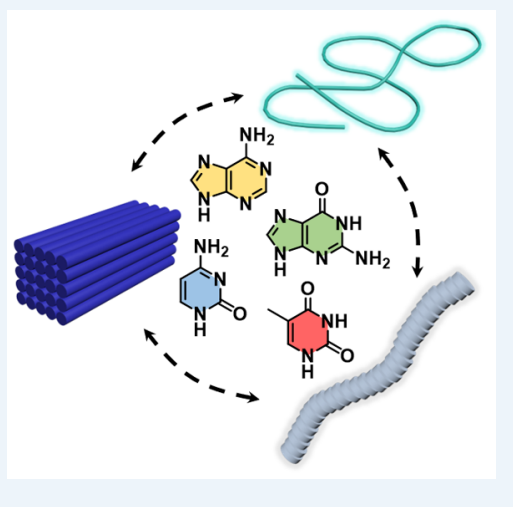

17 he cell and the cellular environment are an inspiration 18 for many scientists. Complex tasks are performed in 19 highly crowded spaces that are often prone to 20 interference, yet these processes are regulated with high spatial 21 and temporal control. For many years, scientists have 22 attempted to understand cellular processes, and despite great 23 success, much is yet to be discovered. In order to understand 24 these complex phenomena and to improve our ability to 25 manipulate them, artificial mimics are currently being 26 developed, although they are simplistic compared to those 27 occurring naturally (Figure 1 ). Within synthetic biology, where 28 biological components are used to build novel functional 29 biological systems, the field of DNA nanotechnology is rapidly 30 expanding.

31 The structure of DNA opens up numerous possibilities in 32 the biomedical field, due to DNA's ability to bind sequences 33 specifically and programmably through canonical Watson34 Crick base pairing of its nucleobase substituents. DNA is able 35 to be manipulated, for example, into various morphologies and 36 implemented as complementary cross-linkers to form hydro37 gels. ${ }^{1,2}$ Because these interactions depend on multivalent 38 noncovalent hydrogen bonding, reversibility is readily 39 obtained, for example, by changes in salt concentration, 40 temperature, or complementary deoxyoligonucleotide displace41 ment strands. In order to retain the programmable properties 42 of DNA with high spatial precision, DNA by itself has also 43 been used as a building block to construct various elegant but 44 complex structures known as DNA origami.

45 Although DNA holds great potential, the negative charge on 46 the structure restricts cell internalization, and the backbone is 47 prone to hydrolysis and enzymatic degradation. In order to increase stability and to include new functionalities that are not 48 obtained by DNA alone, a wide variety of DNA-based 49 materials have been developed. These materials incorporate a 50 range of bioconjugates, from nucleobases and nucleosides that 51 include the universal DNA hydrogen-bonding recognition 52 array to long deoxyoligonucleotides that also have regular 53 helicity due to the presence of the phosphate backbone. ${ }^{3-5} 54$

In this Perspective, we highlight the challenges and 55 opportunities of DNA-based materials, with a focus on the 56 fields of covalent polymers, DNA origami, and supramolecular 57 polymers. It has become clear that these fields complement 58 each other and could potentially be combined in next- 59 generation DNA-hybrid materials for future biomedical 60 applications (Figure 2). We review the steps already taken 61 toward the development of DNA-hybrid materials and the 62 potential for future developments.

DNA-Based Covalent Polymers. Covalent polymers are 64 synthesized by the polymerization of small monomers (e.g., 65 acrylates and methacrylates), which is initiated by radicals, 66 cations, or anions. Aside from homopolymers, random 67 copolymers and block copolymers can be fabricated by seeding 68 monomers with different substitutions. Covalent polymer- 69 ization is ideal to form long and stable structures and is, hence, 70 highly scalable (Figure 2). Typically, the molecular weight 71 distribution, polydispersity, and chain-end functionality can be 72 controlled using living radical polymerization techniques. Aside 73 from controlling structural properties, the degradation of 74 covalent polymers can be tuned by the incorporation of ester 75 groups. ${ }^{6}$ Diverse DNA-based covalent polymers have already 76 


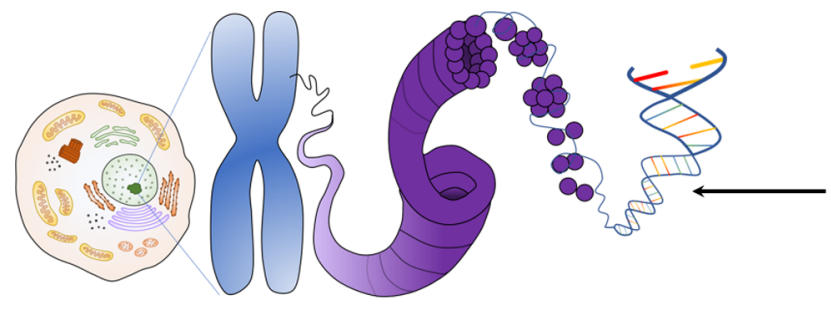

Natural structures

-complex-

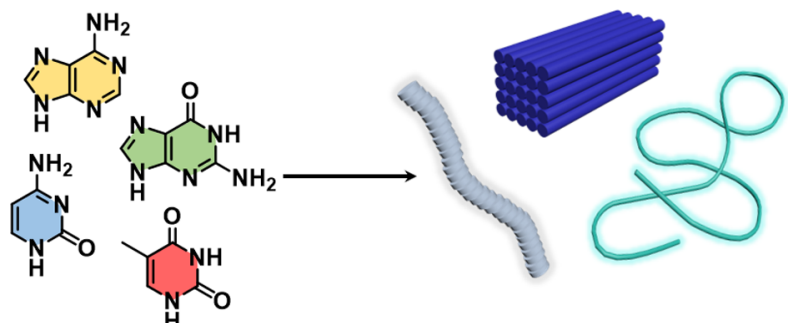

DNA as

building block
Human-made

-simple with emergent properties-

Figure 1. Complex natural structures as compared to simple, human-made artificial materials.

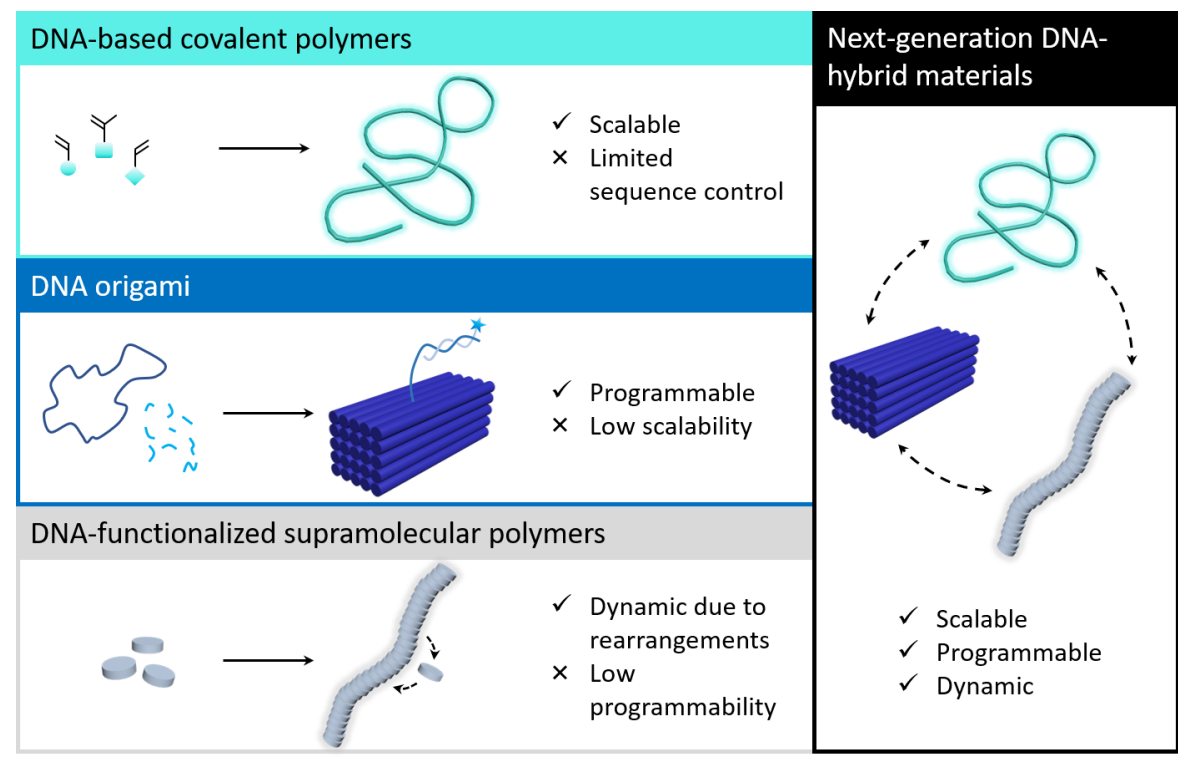

Figure 2. DNA-based covalent polymers, DNA origami, and DNA-functionalized supramolecular polymers with their advantages and disadvantages. Merging these fields enables one to incorporate the best properties of all three fields into next-generation DNA-hybrid materials.

77 been developed, ranging from single nucleobases to 78 oligonucleotide grafts. $^{3-5}$ The latter includes designs where 79 oligonucleotide sequences are grafted to, from, or through, 80 creating polymers with various architectures.

81 Although covalent polymers show promising developments, 82 some living radical polymerization techniques, including atom 83 transfer radical polymerization (ATRP), require a metal 84 catalyst to initiate the polymerization reaction. These metals 85 can potentially chelate with nucleotides, and remnants are 86 difficult to remove, which may result in cell toxicity in 87 biomedical applications. Moreover, overall synthesis yields 88 tend to be low due to solubility issues, and only partial 89 functionalization is obtained when using a postpolymer 90 modification strategy. In order to circumvent these limitations, 91 reversible addition-fragmentation chain-transfer (RAFT) 92 polymerization strategies can be utilized with prefunctionalized 93 protected nucleotide monomers. Using this technique, bulky 94 biofunctional groups may still cause steric hindrance during 95 polymerization; however, this effect can be minimized by 96 tuning the linker length.

97 A major disadvantage of covalent polymers is that it is 98 difficult to achieve spatial functionalization and to implement 99 dynamics that allow local rearrangements in the backbone.
Moreover, covalent polymers tend to form random coils or 100 single-chain nanoparticles in solution, shielding biofunctional 101 group availability and, hence, sterically hindering host-guest 102 binding.

Regardless of these intrinsic shortcomings, DNA-based 104 covalent polymers have already found applications in sensors, 105 DNA and drug delivery, cancer therapy, stimuli-response 106 hydrogels, logic gates, and actuators. ${ }^{3}$ Recent efforts have 107 demonstrated free radical polymerization in situ within living 108 cells, enabling intracellular manipulation, control, and tracking, 109 paving the way toward unprecedented biomedical applica- 110 tions. Although exciting results have been obtained, all of 111 these applications remain in their infancy due to low signal 112 sensitivity and slow response rate, limiting accurate quantifi- 113 cation and single-base mismatch discrimination. Our under- 114 standing of the interactions between DNA-based covalent 115 polymers and cells, including other biomolecules, cellular 116 components, and vesicles, is incomplete, and further research 117 will be required to extend their application potential.

118

DNA Origami Noncovalent Structures. Hierarchical 119 structure and spatial control is readily obtained with DNA 120 origami (Figure 2). ${ }^{1}$ By folding a long bacteriophage-derived 121 single-stranded DNA into a desired structure, any two- 122 
123 dimensional (2D) or three-dimensional (3D) morphology can 124 be fabricated by including hundreds of short staple strands 125 forming crossovers by so-called Holliday junctions. Nanometer 126 spatial control over functionalized groups can be implemented 127 by designing staple strands with a "toehold". Functionalized 128 moieties with a complementary sequence to this toehold can 129 then be coupled. By tuning the length of the complementary 130 sequence, functionalized moieties can be tightly anchored or 131 be dynamic and, hence, in equilibrium with the aqueous 132 solvent. This tunability and binding equilibrium ( $k_{\text {on }}$ and $\left.k_{\text {off }}\right)$ 133 has shown to improve fluorescence microscopy down to sub134 nanometer resolution, identified as DNA point accumulation 135 for imaging in nanoscale topography (DNA-PAINT) super136 resolution microscopy. ${ }^{8}$

\section{Hierarchical structure and spatial con- trol is readily obtained with DNA origami.}

137 DNA-origami-based structures have already found promising 138 applications from molecular robots and nanoreactors to drug 139 delivery and cell internalization. ${ }^{1,9}$ High control over the 140 dimensions, functionality, and manipulation, however, comes 141 with the sacrifice of poor scalability. ${ }^{10}$ Furthermore, hundreds 142 of staple strands are required for one origami structure, which 143 means that building more complex multicomponent structures 144 and preparing larger quantities is prone to high costs and 145 assembly errors. Lastly, error quantification and structural 146 purification are still challenging. Currently, various techniques 147 are being developed to overcome this limitation, including 148 hierarchical assembly using interaction motives and multi149 scaffold designs. ${ }^{11,12}$ Recently, Dietz et al. developed rings and polyhedra that span megadalton $(\mathrm{MDa})$ and even gigadalton 150 (GDa) sizes, approaching dimensions of $450 \mathrm{~nm}$ in diameter 151 (Figure 3A), ${ }^{11}$ illustrating the potential of this approach. $152 \mathrm{f3}$

DNA-Functionalized Supramolecular Polymers. Mov- 153 ing closer to life-like materials, supramolecular polymers mimic 154 natural processes in the sense that they are modular, tunable, 155 adaptable, and responsive. ${ }^{13}$ Due to a combination of weak 156 noncovalent interactions, usually in the form of hydrophobic 157 effects, hydrogen bonding, and $\pi-\pi$ interactions, the small 158 molecular building blocks self-assemble into one-dimensional 159 (1D) fibrous structures in which the monomers can migrate 160 both within and between fibrous assemblies, providing these 161 structures with intrinsic dynamic properties (Figure 2). ${ }^{14} 162$ These structures require a precisely balanced hydrophilic-to- 163 hydrophobic ratio; otherwise, spherical micelles are obtained. 164 Moreover, extending monomers with bulky biofunctional 165 groups, such as peptides and proteins, can interfere with self- 166 assembly due to an imbalance in the hydrophilic-to-hydro- 167 phobic ratio and/or noncovalent interactions, such as hydro- 168 phobic and hydrogen bonding. Aggregate stability can be 169 tuned by improving noncovalent interactions and, hence, the 170 packing between monomers. However, monomers could 171 potentially be released from their supramolecular polymeric 172 support by pulling forces exerted by cells.

Moving closer to life-like materials, supramolecular polymers mimic natural processes in the sense that they are modular, tunable, adaptable, and responsive.
A
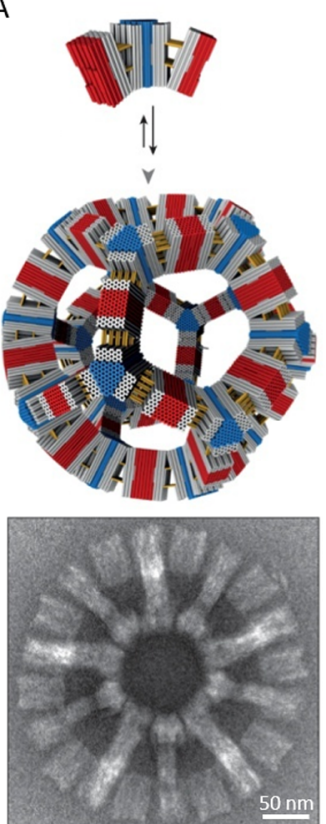
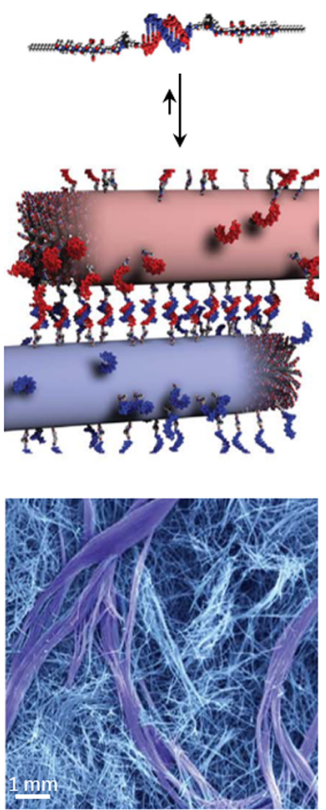
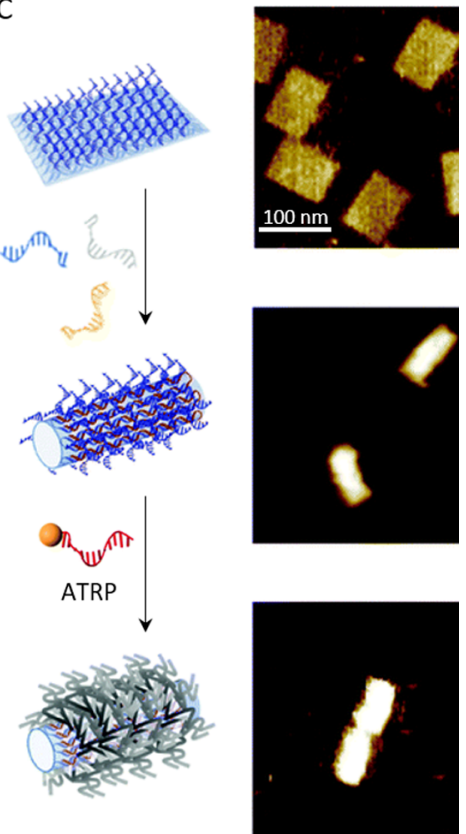

Figure 3. DNA-based nanomaterials. (A) DNA origami structures are assembled into a 1.2 GDa hierarchical polyhedral using interaction motives. Adapted and modified with permission from ref 11. Copyright 2017 Springer Nature. (B) Supramolecular DNA-peptide amphiphiles self-assemble into reversible intertwined superstructures. Adapted and modified with permission from ref 17 . Copyright 2018 American Association for the Advancement of Science. (C) DNA platform is folded into a DNA tube upon which polymers are grafted using atom transfer radical polymerization. Corresponding atomic force microscopy images are depicted to the right. Adapted and modified with permission from ref 29. Copyright 2018 The Royal Society of Chemistry. 
174 Interesting supramolecular polymers containing deoxyoligo175 nucleotides have found applications in tissue engineering, 176 protein recruitment, and logic gates, showing life-like and 177 responsive properties not found in covalent polymers and 178 DNA origami. ${ }^{15}$ Häner and co-workers observed that supra179 molecular assemblies functionalized with single nucleotides 180 formed $2 \mathrm{D}$ sheets as opposed to $1 \mathrm{D}$ fibers when longer 181 deoxyoligonucleotide linkers were incorporated. ${ }^{16}$ This finding 182 highlights that molecular design plays a significant role in the 183 aggregate morphology that supramolecular polymers form. The 184 importance of molecular design was also illustrated in extensive 185 studies by Stupp and co-workers using complementary DNA186 peptide amphiphiles. ${ }^{17}$ Spherical micelles obtained by the 187 individual components formed rapid intertwined super188 structures upon mixing (Figure $3 \mathrm{~B}$ ) that were shown to be 189 reversible upon the addition of displacement deoxynucleotide 190 strands. In supramolecular polymers, the intrinsic dynamic 191 property aids in backbone rearrangements for increased order 192 and optimal host-guest binding. However, as a result, 193 sequence control and, hence, programmability are difficult to 194 achieve. In addition, because supramolecular polymerization is 195 prone to fall into kinetic traps, protocols that govern self196 assembly are critical and fundamental understanding of their 197 pathway complexity is required. ${ }^{18,19}$

198 Toward DNA-Hybrid Materials. The field of DNA 199 nanotechnology has been advanced by combining major 200 developments in the fields of biology, physics, chemistry, 201 material science, and computer science. ${ }^{20,21}$ Incorporating 202 small organic and inorganic molecules into DNA origami 203 structures has already improved stability and rigidity, as 204 compared to DNA-only counterparts. ${ }^{22}$ In addition, fewer 205 DNA staple strands are required, enabling the creation of more 206 complex structures that are better suited for in vivo 207 applications. As well as the four standard DNA nucleobases, 208 a handful of DNA derivatives have been developed that show 209 improved stability and lower immunogenicity. ${ }^{23,24}$ This 210 development increases the DNA toolbox and opens up further 211 possibilities to build structurally diverse materials with highly 212 programmable and spatially addressable properties.

213 By taking advantage of the self-assembly of amphiphilic 214 structures, Sleiman and co-workers developed nanocages with 215 grafted polymers. ${ }^{25}$ Upon self-assembly, these cages formed 216 higher-order structures containing hydrophobic cores, in which 217 the cage aggregation number was dependent on the hydro218 phobic chain length. Therapeutic molecules or nanoparticles 219 could be encapsulated within these hydrophobic cores, 220 nanocages decorated with cholesterol can form nanopores in 221 membranes, and DNA patterns can also be printed on gold 222 nanoparticles using sequence-grafted DNA nanostructures. ${ }^{26,27}$ 223 More complex hybrid materials could potentially be 224 achieved by merging the fields of covalent polymers, DNA 225 origami, and supramolecular polymers. Interesting hybrid 226 DNA-based materials have already been developed in the 227 group of Tanja Weil. Using a DNA origami template, polymers 228 were grafted in a pattern, which enabled the fabrication of 229 patterned polymers with nanometer resolution. ${ }^{28}$ This 230 approach was extended to fabricate polymer-coated DNA 231 tubes (Figure 3C) containing DNAzymes in the interior of the 232 tube. The obtained polymer tube was shown to support 233 catalytic activity and to provide protection against nuclease 234 digestion of these DNAzymes. ${ }^{29}$ This approach opens up the 235 possibility of delivering labile cargo and spatially functionaliz236 ing either the inside or outside of DNA tubes with nanometer precision; such high-order control over covalent polymers has 237 not been achieved elsewhere. These exciting examples set the 238 stage for nanometer hierarchically controlled micro- and 239 macrostructures that have not been possible until now.

240

\section{CONCLUSIONS AND PROSPECTS}

241

DNA-based covalent polymers, DNA origami, and DNA- 242 functionalized supramolecular polymers have shown emergent 243 properties in a wide variety of applications. Yet these materials 244 are still simple compared to naturally occurring biomolecules, 245 restricting their potential for sophisticated applications. 246 Knowledge and techniques from multiple fields are required 247 to design more complex and functional DNA-hybrid materials 248 to advance the field forward. Detailed fundamental under- 249 standing of nucleotide-material interactions is also essential to 250 equip polymers with full DNA functionalities. Novel exciting 251 DNA-hybrid materials are already emerging that enable more 252 control over nanometer spatial resolution, moving the field 253 closer to real-life properties and unprecedented biomedical 254 applications, such as in designing artificial materials that can 255 direct organogenesis, drug delivery, enzymatic activity, and 256 gene editing with remarkably high precision. By merging the 257 fields of covalent polymers, DNA origami, and supramolecular 258 polymers, DNA-hybrid materials that are scalable, program- 259 mable, and possess intrinsic dynamic properties can potentially 260 be realized, providing combined properties that cannot be 261 obtained by any of these materials individually.

\section{AUTHOR INFORMATION}

\section{Corresponding Author}

*E-mail: amanda.ellis@unimelb.edu.au.

Simone I.S. Hendrikse: 0000-0002-8902-613X

Sally L. Gras: 0000-0002-4660-1245

Amanda V. Ellis: 0000-0002-0053-5641

Notes

The authors declare no competing financial interest.

\section{ACKNOWLEDGMENTS}

This work was supported by funding of The Australian 273 Research Council (ARC), Discovery Project (DP190100055). 274

\section{REFERENCES}

(1) Hong, F.; Zhang, F.; Liu, Y.; Yan, H. DNA Origami: Scaffolds for 276 Creating Higher Order Structures. Chem. Rev. 2017, 117, 12584- 277 12640 .

(2) Shao, Y.; Jia, H.; Cao, T.; Liu, D. Supramolecular Hydrogels 279 Based on DNA Self-Assembly. Acc. Chem. Res. 2017, 50, 659-668. 280

(3) Peng, L.; Wu, C. S.; You, M.; Han, D.; Chen, Y.; Fu, T.; Ye, M.; 281 Tan, W. Engineering and Applications of DNA-Grafted Polymer 282 Materials. Chem. Sci. 2013, 4, 1928-1938.

(4) Sun, H.; Yang, L.; Thompson, M. P.; Schara, S.; Cao, W.; Choi, 284 W.; Hu, Z.; Zang, N.; Tan, W.; Gianneschi, N. C. Recent Advances in 285 Amphiphilic Polymer-Oligonucleotide Nanomaterials via Living/ 286 Controlled Polymerization Technologies. Bioconjugate Chem. 2019, 287 30, 1889-1904.

(5) Yang, H.; Xi, W. Nucleobase-Containing Polymers: Structure, 289 Synthesis, and Applications. Polymers 2017, 9, 666.

(6) Kamaly, N.; Yameen, B.; Wu, J.; Farokhzad, O. C. Degradable 291 Controlled-Release Polymers and Polymeric Nanoparticles: Mecha- 292 nisms of Controlling Drug Release. Chem. Rev. 2016, 116, 2602- 293 2663. 
295 (7) Geng, J.; Li, W.; Zhang, Y.; Thottappillil, N.; Clavadetscher, J.; 296 Lilienkampf, A.; Bradley, M. Radical Polymerization Inside Living 297 Cells. Nat. Chem. 2019, 11, 578-586.

298 (8) Jungmann, R.; Avendaño, M. S.; Woehrstein, J. B.; Dai, M.; Shih, 299 W. M.; Yin, P. Multiplexed 3D Cellular Super-Resolution Imaging 300 with DNA-PAINT and Exchange-PAINT. Nat. Methods 2014, 11, 301 313-318.

302 (9) Hu, Q.; Wang, S.; Wang, L.; Gu, H.; Fan, C. DNA 303 Nanostructure-Based Systems for Intelligent Delivery of Therapeutic 304 Oligonucleotides. Adv. Healthcare Mater. 2018, 7, 1701153.

305 (10) Pinheiro, A. V.; Han, D.; Shih, W. M.; Yan, H. Challenges and 306 Opportunities for Structural DNA Nanotechnology. Nat. Nanotechnol. 307 2011, 6, 763-772.

308 (11) Wagenbauer, K. F.; Sigl, C.; Dietz, H. Gigadalton-Scale Shape309 Programmable DNA Assemblies. Nature 2017, 552, 78-83.

310 (12) Engelhardt, F. A. S.; Praetorius, F.; Wachauf, C. H.; 311 Brüggenthies, G.; Kohler, F.; Kick, B.; Kadletz, K. L.; Pham, P. N.; 312 Behler, K. L.; Gerling, T.; Dietz, H. Custom-Size, Functional, and 313 Durable DNA Origami with Design-Specific Scaffolds. ACS Nano 314 2019, 13, 5015-5027.

315 (13) Webber, M. J.; Appel, E. A.; Meijer, E. W.; Langer, R. 316 Supramolecular Biomaterials. Nat. Mater. 2016, 15, 13-26.

317 (14) Goor, O. J. G. M.; Hendrikse, S. I. S.; Dankers, P. Y. W.; Meijer, 318 E. W. From Supramolecular Polymers to Multi-Component 319 Biomaterials. Chem. Soc. Rev. 2017, 46, 6621-6637.

320 (15) Wijnands, S. P. W.; Meijer, E. W.; Merkx, M. DNA321 Functionalized Supramolecular Polymers: Dynamic Multicomponent 322 Assemblies with Emergent Properties. Bioconjugate Chem. 2019, 30, 323 1905-1914.

324 (16) Vyborna, Y.; Altunbas, S.; Vybornyi, M.; Häner, R. 325 Morphological Diversity of Supramolecular Polymers of DNA326 Containing Oligopyrenes - Formation of Chiroptically Active 327 Nanosheets. Chem. Commun. 2017, 53, 12128-12131.

328 (17) Freeman, R.; Han, M.; Álvarez, Z.; Lewis, J. A.; Wester, J. R.; 329 Stephanopoulos, N.; McClendon, M. T.; Lynsky, C.; Godbe, J. M.; 330 Sangji, H.; Luijten, E.; Stupp, S. I. Reversible Self-Assembly of 331 Superstructured Networks. Science 2018, 362, 808-813.

332 (18) Tantakitti, F.; Boekhoven, J.; Wang, X.; Kazantsev, R. V.; Yu, 333 T.; Li, J.; Zhuang, E.; Zandi, R.; Ortony, J. H.; Newcomb, C. J.; 334 Palmer, L. C.; Shekhawat, G. S.; de la Cruz, M. O.; Schatz, G. C.; 335 Stupp, S. I. Energy Landscapes and Functions of Supramolecular 336 Systems. Nat. Mater. 2016, 15, 469-476.

337 (19) Mattia, E.; Otto, S. Supramolecular Systems Chemistry. Nat. 338 Nanotechnol. 2015, 10, 111-119.

339 (20) Seeman, N. C.; Sleiman, H. F. DNA Nanotechnology. Nat. Rev. 340 Mater. 2018, 3, 17068.

341 (21) Jia, F.; Li, H.; Chen, R.; Zhang, K. Self-Assembly of DNA342 Containing Copolymers. Bioconjugate Chem. 2019, 30, 1880-1888.

343 (22) Greschner, A. A.; Toader, V.; Sleiman, H. F. The Role of 344 Organic Linkers in Directing DNA Self-Assembly and Significantly 345 Stabilizing DNA Duplexes. J. Am. Chem. Soc. 2012, 134, 1438234614389.

347 (23) Wickstrom, E. DNA and RNA Derivatives To Optimize 348 Distribution and Delivery. Adv. Drug Delivery Rev. 2015, 87, 25-34. 349 (24) Pinheiro, V. B.; Holliger, P. Towards XNA Nanotechnology: 350 New Materials from Synthetic Genetic Polymers. Trends Biotechnol. 351 2014, 32, 321-328.

352 (25) Chidchob, P.; Edwardson, T. G. W.; Serpell, C. J.; Sleiman, H. 353 F. Synergy of Two Assembly Languages in DNA Nanostructures: Self354 Assembly of Sequence-Defined Polymers on DNA Cages. J. Am. 355 Chem. Soc. 2016, 138, 4416-4425.

356 (26) Trinh, T.; Liao, C.; Toader, V.; Barłóg, M.; Bazzi, H. S.; Li, J.; 357 Sleiman, H. F. DNA-Imprinted Polymer Nanoparticles with 358 Monodispersity and Prescribed DNA-Strand Patterns. Nat. Chem. 359 2018, 10, 184-192.

360 (27) Chidchob, P.; Offenbartl-Stiegert, D.; McCarthy, D.; Luo, X.; 361 Li, J.; Howorka, S.; Sleiman, H. F. Spatial Presentation of Cholesterol 362 Units on a DNA Cube as a Determinant of Membrane Protein363 Mimicking Functions. J. Am. Chem. Soc. 2019, 141, 1100-1108.
(28) Tokura, Y.; Jiang, Y.; Welle, A.; Stenzel, M. H.; Krzemien, K. 364 M.; Michaelis, J.; Berger, R.; Barner-Kowollik, C.; Wu, Y.; Weil, T. 365 Bottom-Up Fabrication of Nanopatterned Polymers on DNA Origami 366 by in Situ Atom-Transfer Radical Polymerization. Angew. Chem., Int. 367 Ed. 2016, 55, 5692-5697.

368

(29) Tokura, Y.; Harvey, S.; Xu, X.; Chen, C.; Morsbach, S.; 369 Wunderlich, K.; Fytas, G.; Wu, Y.; Ng, D. Y. W.; Weil, T. Polymer 370 Tube Nanoreactors via DNA-Origami Templated Synthesis. Chem. 371 Commun. 2018, 54, 2808-2811. 